\title{
Infrared thermography study of a confined impinging circular jet
}

\author{
by J.-M. Buchlin and M. Meyers
}

von Karman Institute for Fluid Dynamics, Rhode-Saint-Genèse, Belgium

\begin{abstract}
The convective heat transfer at the impingement of a vertical turbulent circular air jet on a horizontal flat plate is inferred from temperature measurements performed by quantitative infrared thermography. Steadystate experiments are conducted with the heating-thin-foil method. The effects of the jet Reynolds number and the stand-off distance on the thermal exchange coefficient are emphasized. The influence of jet confinement is determined. The IR results agree with published data and are reproduced by numerical simulations performed with the code FLUENT.
\end{abstract}

\section{NOMENCLATURE}

\begin{tabular}{|c|c|c|c|c|c|}
\hline & & & Greek symbols & & Subscripts \\
\hline$d$ & Exit nozzle diameter & $\varepsilon$ & Emissivity factor & a & Ambient \\
\hline$D_{c}$ & Confinement diameter & $v$ & Kinematic viscosity & b & Bulk \\
\hline$h$ & Heat transfer coefficient & & & c & Confinement \\
\hline$k$ & Thermal conductivity & & & cd & Conduction \\
\hline$N u$ & Nusselt number & & & $\mathrm{cv}$ & Convection \\
\hline$r$ & Radial distance & & & $\mathrm{j}$ & Jet \\
\hline $\operatorname{Re}$ & Reynolds number & & & $\mathrm{j}$ & Joule \\
\hline$T$ & Temperature & & & 0 & Impingement \\
\hline$U$ & Velocity & & & ref & Reference \\
\hline$Z$ & Stand-off distance & & & s & Stagnation \\
\hline$Z_{c}$ & Confinement gap & & & $w$ & Wall \\
\hline
\end{tabular}

\section{Introduction}

Impinging fluid jets are widely used in industrial processes where high heat and/or mass transfer rates have to be reached [1]. Typical examples are the annealing of metals, the tempering of glass sheets, the drying of paper and textiles, the cooling of turbine blades and the anti-icing of aircraft. In many of these applications, the jet expands at a temperature different from the ambient or in a confined space. Such circumstances affect the jet development and modify the impingement conditions so that the heat transfer differs from the unconfined-jet situation. The present paper deals with this question in describing an experimental investigation relying on the application of quantitative thermography. The suitability of such a technique to complex flow situations such as impinging jets has already been demonstrated [2-6].

After presenting the main features of the experimental set-up and the procedure followed to collect the thermal data, the paper emphasises the effect of the flow and geometrical parameters on the distribution of the convective heat transfer coefficient. Finally, the experimental observations are compared to numerical simulations to appraise the applicability of CFD codes to such a flow problem.

\section{Experimental set-up}

The experimental test set-up is sketched in figure 1. It consists of a horizontal flat plate made of a thin copper foil , $35 \mu \mathrm{m}$ thick, machined to produce a spiral circuit fixed on an epoxy sheet, 1.5 $\mathrm{mm}$ thick. The thin foil is heated by Joule effect. The jet is obtained by forcing dry compressed air 
through an aerodynamic Disa nozzle with an exit diameter of $0.012 \mathrm{~m}$. The jet is oriented vertically downward and a heat exchanger controls its temperature. Two types of confinement are tested. In the case of confinement $\mathbf{I}$, the jet spreads in a cylindrical Plexiglas chamber closed at the top at illustrated in figure 1 and shown by the photograph on figure 2. It is characterised by the chamber diameter $D_{c}$ and the air gap of the vent $Z_{C}$ between the heated target plate and the cylindrical chamber.

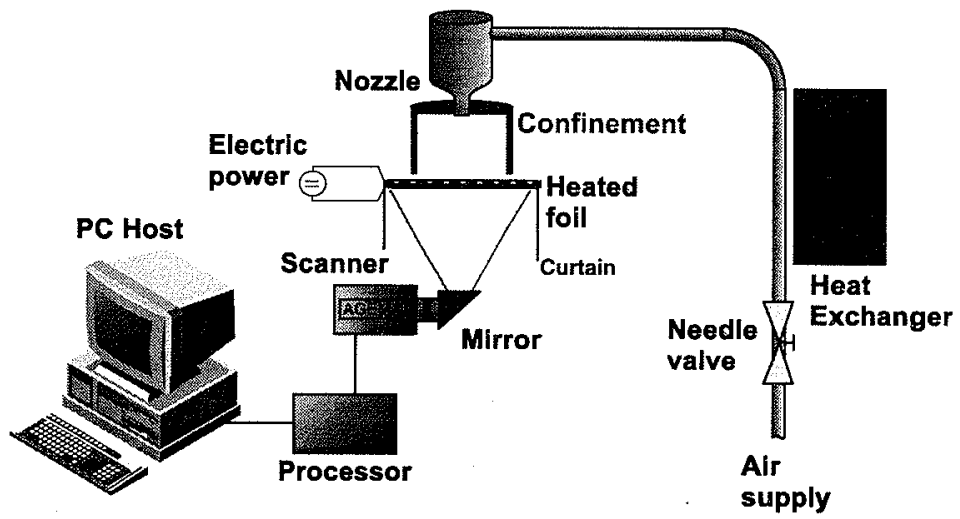

Fig. 1. Schematic of the test set-up
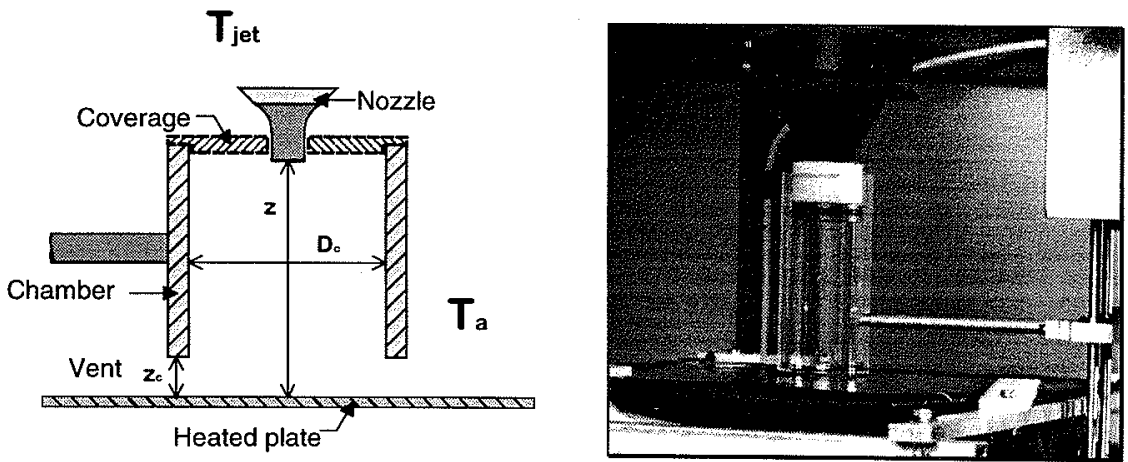

Fig. 2. Close view of the nozzle confinement

In confinement II the vertical chamber is removed and only the covering plate is used. In such configuration $Z_{d} d d=Z / d$. Both faces of the foil are coated with a black paint of emissivity $\varepsilon_{w}=0.90$ -to improve the thermography measurements and allow correction for radiation. The rear surfaco, viewed by the IR scanner, is surrounded by a black plastic curtain that minimizes heat losses by natural convection at the edges. The IR scanner is the AGEMA Thermovision 900 system with a $\mathrm{HgCdTe}$ detector sensitive in the $8-12 \mu \mathrm{m}$ wavelength range and cooled by liquid nitrogen. The measurable temperature range is $-30^{\circ} \mathrm{C}$ to $1500^{\circ} \mathrm{C}$ with an announced thermal sensitivity of 0,1 ${ }^{\circ} \mathrm{C}$. The camera is equipped with a standard optical set-up $\left(10^{\circ}\right.$ vertical $\times 5^{\circ}$ horizontal) giving an instantaneous field of view (IFOV) of 0.76 mrad. The camera scans the flat plate via a 45 -degreo mirror leading to a spatial resolution of 1 pixel per millimetre. 


\section{Data restoration}

Calibration of the camera is performed by plotting the thermal unit TU versus the plate temperature measured by a thermocouple flush-mounted on the top surface (jet face) of the target. In the working temperature range $20-80^{\circ} \mathrm{C}$, the calibration curve $T$-TU can be approximated by a quadratic polynomial.

The scanning of the heated flat plate without jet (the situation corresponding to radiative exchange and pure natural convection where the convective heat transfer coefficient is almost constant over the surface) points out the good axisymmetry of the heating but reveals a non-uniform radial distribution as shown in figure 3 . The minimum value at the centre results from the heat loss by conduction through the electrical supply cable. To account for such a non-uniformity during thermogramme restoration, the total heat flux is systematically multiplied by a weighting function $f(r)$ fitting the distribution plotted in figure 3.

The convective heat transfer coefficient is inferred from the temperature of the wall $T_{w}(r)$ by application of the Newton law:

$$
h(r)=\frac{q_{c v}(r)}{T_{w}(r)-T_{\text {ref }}}
$$

where $T_{\text {ref }}$ is a reference temperature taken equal to the jet temperature $T_{j}$ in the present investigation.

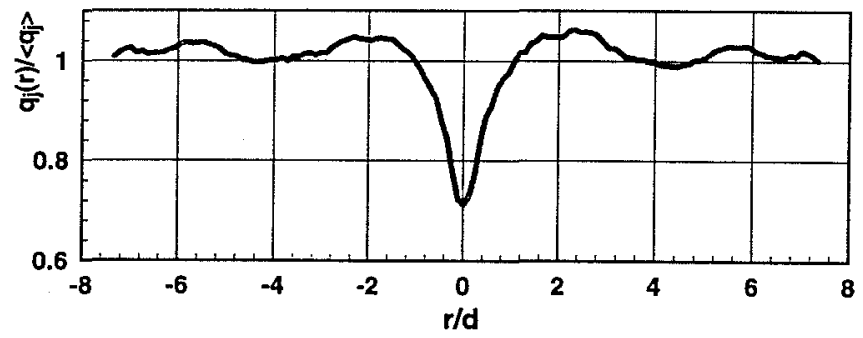

Fig. 3. Heat flux distribution without jet.

The convective heat flux $q_{c y}$ is calculated by subtracting the contribution of the radiation $q_{\mathrm{rad}}$ and lateral conduction $q_{c d}$ from the Joule heating $q_{J}[4-5]$. In such a thin-foil design, the thermal losses by conduction (copper and epoxy) are negligible $(\leq 1 \%)$, while the heat loss by radiation does not exceed $5 \%$ of the total flux.

The net result of the correction during restoration is shown in figure 4 where the radial distribution of the Nusselt number $N u=h d / k_{f}$ is plotted. The correction for the distortion in the wall heat flux is more important than the correction for the heat losses.

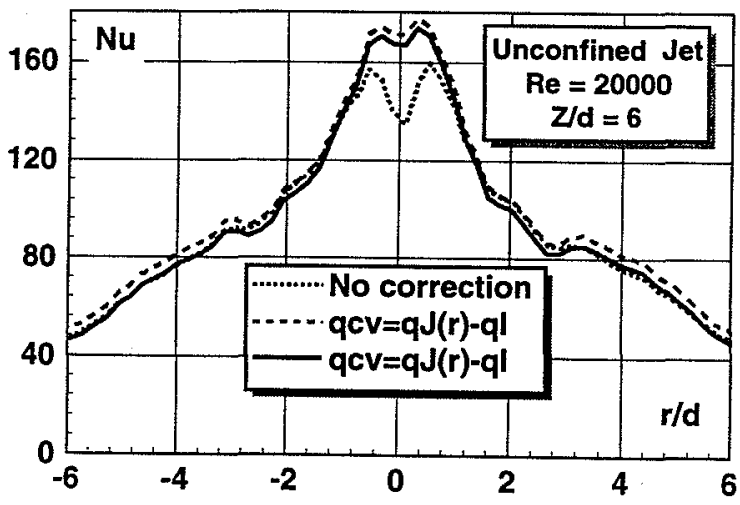

Fig. 4. Restoration effect on the Nu-distribution 


\section{Typical results}

Unconfined jet tests are carried out by varying the jet Reynolds number $R e=U_{j} d / v$ from 5000 to 30000 and normalised stand-off distance $Z / d$ from 2 to 17. Typical results are shown in figure 5.

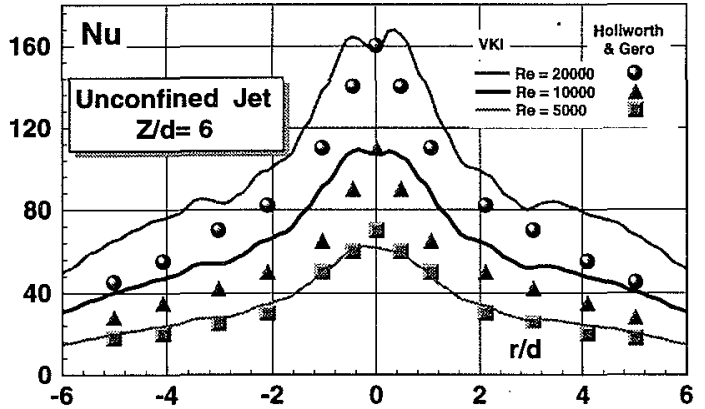

Fig. 5. Radial Nu-distribution: effect of the Reynolds number.

They reproduce the experiments conducted by Hollworth \& Gero [7] who apply a microfoil-heat-flux-sensor technique to measure the thermal exchange. Good agreement is observed in particular at the stagnation value that can be correlated as following:

$\mathrm{Nu}=\mathrm{C}^{\mathrm{te}} R \mathrm{e}^{0.65}$

Relation (2) indicates that the flow in the stagnation zone is not too far from laminar $\left(R e^{0.5}\right)$. The off-center peak located near $r / d=0.5$ is generally attributed to the rapid change in the radial velocity which occurs in the deflection with displacement from the stagnation point [9]. Another explanation relying upon the presence of a local maximum of turbulence near the centre of the mixing region of the approaching jet has been proposed [7]. The distinct change in the slope of the Nu-distribution near $r / d=2$ at high Reynolds number ( $\mathrm{Re} \geq 20000$ ) reflects the existence of toroidal vortices surrounding the jet which strike the heated plate. For stand-off smaller than 6, such a phenomenon leads to the development of a stronger second off-centre peak of heat transfer, significantly lessened in the present experiments due to the value of $Z / d$.

An averaged stagnation Nusselt number $\mathrm{Nu}_{0}$ defined as the mean coefficient between the stagnation value and the inner peak value when existing (see figure 5) is plotted versus the normalised stand-off distance $Z / d$ in figure 6 . The present IR-data agree very well with the Braughn and Shimizu results obtained through liquid-crystal experiments [8]. They are also compared with CFD simulations performed with the code FLUENT which overpredict by only $10 \%$ the experimental observations. Such an agreement is obtained by using a relatively dense meshing in the impingement region and the RNG turbulence model with the low Reynoldsnumber option. The maximum of the stagnation heat-transfer rate found at $Z / d=6$ occurs when the axial increase of turbulence in the jet due to en-

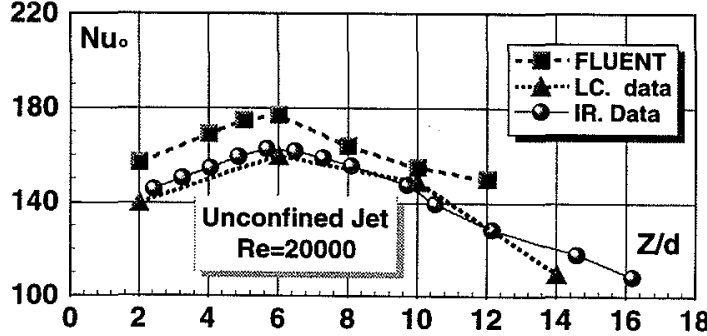

Fig. 6. Effect of the normalised stand-off distance on the stagnation $\mathrm{Nu}_{0}$-number trainment does not compensate for the fall in the jet velocity. In other words, that corresponds to the end of the potential core and the arrival of turbulence from the shear layer at the stagnation zone.

As shown in figure 7 , the confinement causes a general reduction in the heat transfer coefficient based on the jet temperature as compared to the unconfined-jet case. Such a behaviour is the result of the recirculating flow that is established in the chamber as reproduced by the 
FLUENT simulations displayed in figure 8 . The bulk temperature $T_{b}$ of the fluid recirculating in the chamber becomes higher than the exit jet temperature due to the heat removal. Therefore, at steady-state the heat transfer coefficient drops compared to the unconfined jet situation. Since the driving temperature used in the present definition of the heat transfer coefficient is larger than the more appropriate difference $T_{w}-T_{b}$, smaller $N u$-values are obtained. However, the approach of choosing $T_{\text {ref }}=T_{b}$ involves practical difficulties for engineering application since this temperature is never known a priori. Figure 9 shows that the test-repeatability is good and the numerical predictions are able to reproduce adequately the experimental observations also in the confinement case.

The range of the geometrical parameters considered in figure 7 does not bring firm conclusions about the sensivity of the heat transfer to the confinement size. However, the comparison of the stagnation Nusselt number, found for a gap of $Z_{d} d=0.08$ in figure 9 , to the corresponding value plotted in figure 7 for $Z_{d} d d=0.4$ shows a net decrease of the heat transfer when the vented cross section diminishes.

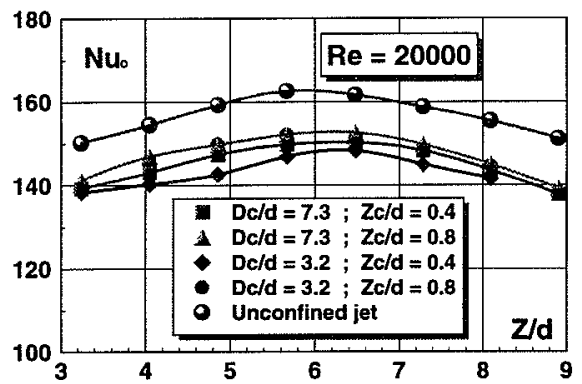

Fig. 7. Effect of the confinement on $\mathrm{Nu}_{0}$

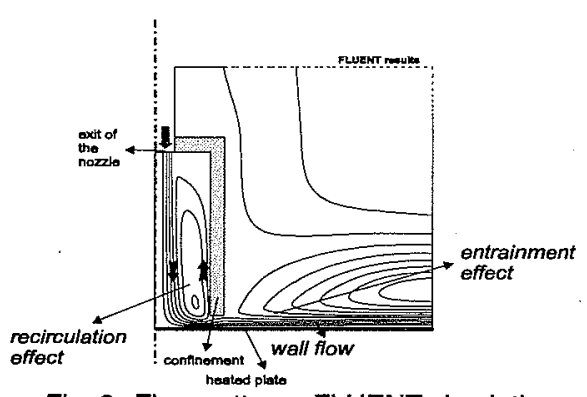

Fig. 8. Flow pattern : FLUENT simulation

The case of confinement II characterised by a gap equals to the stand-off distance as investigated by Lucas et al [10] with liquid crystals (LC) is illustrated by figure 10. In such experiments a long covering plate with $D_{d} d=20$ is maintained at the jet temperature by means of a cooling circuit. The IR data compare well with the LC data; the decrease of the stand-off distance leads to an increase of the confinement effect and provokes a drop of the heat transfer, more pronounced in the impingement region. Again, such a decrease can be associated to the formation of a recirculation bubble close to the jet due to entrainment and more pronounced in the case of small $Z / d$.

\section{Conclusions}

Steady-state measurements of the heat transfer at the impingement of a vertical downward air jet are obtained using the heating-thin-foil method and quantitative infrared thermography. Thermograms' calibration and corrections for non-uniform heating and heat loss are performed. The infrared data agree well with the results obtained either by microfoil-heat-flux sensors or liquid crystals both for the unconfined and confined jet. Comparisons between the IR-data and CFD simulations, performed with the code FLUENT, show that numerical simulations can reproduce the experimental results. The presence of the confinement leads to a reduction of the heat transfer coefficient based on the temperature difference between the heated wall and the air jet. 


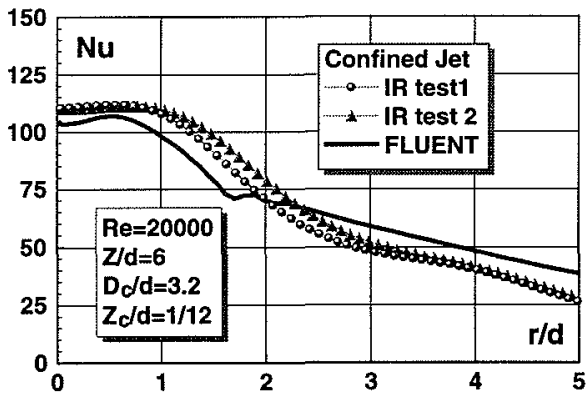

Fig. 9. Comparison between IR. data and FLUENT simulation

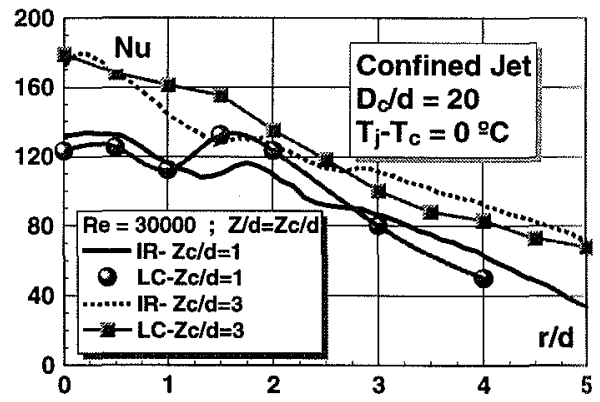

Fig. 10. Nusselt number distribution in a confined jet

\section{REFERENCES}

[1] MARTIN (H). - Heat and Mass Transfer between impinging gas jets and solid surfaces. Adv. Heat Transfer, Vol. 13, 1977, pp. 1-60.

[2] - BUCHLIN (J-M). - Thermography application to heat transfer of impinging jets in "Flow visu* alization and digital image processing", Lecture -Series 1986-09, von Karman Institute for Fluid Dynamics, Rhode Saint Genèse, Belgium, June 1986, pp. 13-22.

[3] BUCHLIN (J-M). - Digital image processing technique applied to fluid dynamic problems. AGARD PEP $67^{\text {th }}$ Symp. on Advanced Instrumentation for Aero Engine Components, Philadelphia, USA, May 1986.

[4] BUCHLIN (J-M) and DUBOIS (M). - Heat transfer of impinging multijet system. An application of the quantitative thermography. Quantitative Infrared Thermography QIRT 92, EUROTHERM Seminar 27, Châtenay-Malabry, France, July 7-9, 1992, p. 117-120.

[5] BUCHLIN (J-M); PRETREL (H); PLANQUART (P); LANGER ( H); THIRY (F).-Infrared thermography study of a thermal anti-icing system. Eurotherm No 42 "Quantitative Infrared thermography" QIRT 94, Sorento, Italy, August 1994. VKI PP 1994-54.

[6] BUCHLIN (J-M); PRETREL (H); PLANQUART (P); Etude thermographique d'un système de dégivrage à jets d'air. Société française des Thermiciens Journée d'Etude sur la Thermographie Infrarouge, Paris, December 7, 1994. VKI PP 1995-06.

[7] HOLWORTH (B.R.) and GERO ( L.R.); Entrainment effects on impingement heat transfer: Part II- Local heat transfer measurements. Journal of Heat Transfer, Transactions of the ASME, Vol.107, Nov. 1985, pp 910-915.

[8] BAUGHN (J.W.) and SHIMIZU (S.): Heat transfer measurements from a surface with uniform heat flux and impinging jet. Journal of Heat Transfer, Transactions of the ASME, Vol.111, Nov. 1989, pp 1096-1098.

[9] JUMBUNATHAN (K.); LAI (E.); MOSS (M.A.); BUTTON (B.L.): A review of heat transfer data for single circular jet impingement. Int. J. Heat and Fluid Flow. Vol. 13, No 2., June 1992.

[10] LUCAS (M.G.); IRELAND (P.T.); WANG (Z.); JONES (T.V.): Fundamental studies of impingement cooling thermal boundary conditions. Heat Transfer and Cooling in Gas Turbines, AGARD CP-527, Antalya, Turkey 12-16 October 1992. 